\title{
Progressive anterior knee pain associated with patellar instability in a 57-year-old father and his daughter
}

\author{
Ali Al Kaissi • Klaus Klaushofer • Franz Grill
}

Published online: 18 March 2010

(C) ISS 2010

Keywords Small patella syndrome · Patellar instability .

Osteoarthritis $\cdot$ Radiographs

\section{Diagnosis}

Patellar instability in a family with small patella syndrome

\section{Discussion}

Small patella syndrome is a generalized bone dysplasia with diagnostic features in the pelvis and knees and subtle changes in other parts of the skeleton. Scott and Taor [1] reported 12 individuals from an autosomal dominant pedigree with small or absent patellae. Seven individuals also had abnormalities of the pelvis or upper femora consisting of coxa vara, hypoplasia of the lesser trochanter, and abnormal ossification of the ischio-pubic junction. Bernhang and Levine [2] reported bilateral absence of the patella detected in a 14-year-old child because of a cystic swelling over one knee. This revealed a clear fluid and the

The case presentation can be found at doi: 10.1007/s00256-010-0880-x.

A. Al Kaissi $(\bowtie) \cdot$ K. Klaushofer

Ludwig Boltzmann Institute of Osteology at the Hanusch Hospital of WGKK and AUVA Trauma Centre Meidling,

4th Medical Department, Hanusch Hospital,

Heinrich Collin Strasse 30,

1140 Vienna, Austria

e-mail: ali.alkaissi@osteologie.at

A. Al Kaissi · F. Grill

Paediatric Department, Orthopaedic Hospital of Speising,

Speisinger Strasse 109,

Vienna 1130, Austria cyst had to be surgically excised. Radiologically, other than the absent patellae, there were no osseous lesions. The patient's brother was similarly affected. Bongers et al. [3] provided the first evidence for the crucial role of human TBX4 gene in the skeletal development of the patella, pelvis, and feet. They identified the heterozygosity of TBX4 mutations in SPS patients, together with the similar skeletal phenotype of animals lacking $T b \times 4$.

Patellar stability relies on the limb alignment, the osseous architecture of the patella and the trochlear, the integrity of the soft tissue constraints, and the interplay of the surrounding muscles [4]. Previous reports described trochlear dysplasia as an abnormality of the shape and depth of the trochlear groove, mainly in its proximal extent. This was defined radiologically by Dejour et al. [5] on the basis of the crossing sign (croisement). If the line of the trochlear floor crosses the anterior border of one or both condyles, the trochlea is said to be flat at that level. The same feature was described by Grelsamer and Tedder [6] and called the lateral trochlear sign. Trochlear dysplasia was further classified by Dejour and Locatelli [7] and Tavernier and Dejour [8] on the basis of the CT morphology of the index scan and the direct lateral radiograph.

Small patella syndrome should be differentiated from the more common and better-known nail patella syndrome. Nail patella syndrome is characterized by bilateral dysplastic patellae, glaucoma, and nephropathy. Iliac horns are present in only approximately $70-80 \%$ of cases; they represent a pathognomonic radiographic sign [9]. Additional skeletal abnormalities include talipes equinovarus, a malformed capitellum of the radius, and fibular aplasia. The nails are abnormal from birth and are either absent or small and longitudinally ridged. 
The family described here presented with distinctive radiographic features. Figure 1a in the case presentation, a lateral knee radiograph, showed trochlear dysplasia, including the crossing sign, supratrochlear spur, and double contour (a hypoplastic medial facet). Figure 1b, a skyline view, showed lateral tracking of the patella with trochlear dysplasia and lateral patellar compression syndrome of the patello-femoral joint, especially of the left knee with subchondral degenerative changes (this is a common feature in patients with lateral tilted patella, when there is more pressure at the lateral facet). This is typical of osteoarthritis of the patello-femoral joint. Strikingly, there is hypoplasia of the medial condyle and the failure of formation of a femoral notch, more marked on the right side. In Fig. 2a, the skyline view showed dysplasia and subluxation of the patellae and aplasia of the trochlear groove bilaterally and in Fig. $2 \mathrm{~b}$ the transverse 3D double echo steady-state gradient-echo MRI showed a flat trochlear groove (arrows) and a hypoplastic/subluxated patella.

Finally, we wish to stress that patello-femoral dysplasia and patello-femoral osteoarthritis are typical manifestations in association with a small patella.

\section{References}

1. Scott JE, Taor WS. The "small patella" syndrome. J Bone Joint Surg B. 1979;61:172-5.

2. Bernhang AM, Levine SA. Familial absence of the patella. J Bone Joint Surg A. 1973;55:1088-90.

3. Bongers EMHF, Duijf PH, van Beersum SE, Schoots J, Van Kampen A, Burckhardt A, et al. Mutations in the human TBX4 gene cause small patella syndrome. Am J Hum Genet. 2004;74:1239-48.

4. Van Huyssteen AL, Hendric MRG, Barnett AJ, Wakeley CJ, Eldridge JDJ. Cartilage-bone mismatch in the dysplastic trochlea: an MRI study. J Bone Joint Surg [Br]. 2006;81-B:688-91.

5. Dejour H, Walch G, Nove-Josserand L, Guier C. Factors of patellar instability: an anatomic radiographic study. Knee Surg Sports Traumatol Arthrosc. 1994;2:19-26.

6. Grelsamer RP, Tedder JL. The lateral trochlear sign: femoral trochlear dysplasia as seen on a lateral view roentgenograph. Clin Orthop. 1992;281:159-63.

7. Dejour D, Locatelli E. Patellar instability in adults. Surg Tech Orthop Traumatol. 2001;55:1-6.

8. Tavernier T, Dejour D. Knee imaging: what is the best modality? J Radiol. 2001;82:387-405. in French.

9. Banskota AK, Mayo-Smith W, et al. Nail-patella syndrome (hereditary onycho-osteodysplasia) with congenital absence of the fibulae. Skeletal Radiol. 1989;18:318-21. 